\title{
Structure of Neurosecretory Cells with Immunoreactive Diapause Hormone and Pheromone Biosynthesis Activating Neuropeptide in the Silkworm, Bombyx mori
}

\author{
Toshio Ichikawa*, Koh Hasegawa ${ }^{1 * *}$, Isamu Shimizu ${ }^{1}$, \\ Kenji Katsuno ${ }^{2}$, Hiroshi Kataoka ${ }^{2}$ and Akinori Suzuki ${ }^{2}$ \\ Department of Biology, Faculty of Science, Kyushu University, Fukuoka \\ 812-81, ${ }^{1}$ Center for Ecological Research, Kyoto University, Kyoto \\ 606, and ${ }^{2}$ Department of Applied Biochemical Chemistry, \\ Faculty of Agriculture, The University of Tokyo, \\ Tokyo 113, Japan
}

\begin{abstract}
Immunocytochemistry of diapause hormone (DH) or pheromone biosynthesis activating neuropeptide (PBAN) revealed three clusters of neurosecretory cells present on the ventral midline of the suboesophageal ganglion (SG) of the silkworm: there were 4 cells in the anterior, 6 cells in the medial, and 2 cells in the posterior cluster. Intracellular injection of Lucifer Yellow into an anterior or a medial cell revealed that the cell has a dendritic arborization at the anterior region of the SG and that it projects an axon to the corpus cardiacum (CC) via a branch of the maxillary nerve and an associated nerve of the $\mathrm{CC}(\mathrm{NCC}-\mathrm{V})$. A dye-filled posterior cell in the larva projects bilaterally-symmetric dendritic branches to the anterior half of the SG that is to expand laterally to fuse the brain after pupation. The axon of the cell, passing through the brain, enters the NCC 3 to spread varicose terminal branches in the CC and associated nerves of the $\mathrm{CC}$.
\end{abstract}

\section{INTRODUCTION}

Neurosecretory cells in the suboesophageal ganglion (SG) of lepidopteran insects secrete several distinct neurohormones. In the silkworm Bombyx mori, at least two peptide hormones originate from the SG. One is diapause hormone (DH) that acts on the developing ovary to induce diapause eggs $[5-7,9]$, and the other is pheromone biosynthesis activating neuropeptide (PBAN) that stimulates the production of sex pheromone in the pheromone glands of virgin females [1]. DH and PBAN are 24 and 33-34 amino acid peptides, respectively, with a common C-terminal sequence, FXPRLamide $[15,18,19]$.

The cloning and characterization of cDNA for PBAN or DH of Bombyx mori showed that a single gene encoded a common precursor polyprotein, from which DH, PBAN and three other FXPRLamide peptides were released through post-translational processing $[16,30]$. cDNA with a similar organization of multiple neuropeptides, including PBAN, has been identified in another moth, Helicoverpa zea [21]. The gene for DH-PBAN precursor was expressed in three groups of neurosecretory cells clustered in the ventral midline of the SG [31]. In Helicoverpa zea, three similar clusters of neurosecretory cells in the SG were visualized by immunocytochemistry of PBAN [17]. Because neuropeptides with a

Accepted September 11, 1995

Received July 28, 1995

* To whom all correspondence should be addressed.

** Present address: Research Laboratory of Applied Biochemistry, Tanabe Seiyaku Co., LTD., 1689 Kashima 3-chome, Yodogawaku, Osaka 532, Japan.
FXPRLamide motif have a myotropic activity [20] and induce integumental pigmentation [23, 24], the three sets of neurosecretory cells may be involved in processes other than diapause induction and pheromone production, and they may functionally differentiate to secrete specific (sets of) neuropeptides at different stages of postembryonic development of the insect. Characterization of anatomical profiles of the cells, including their terminal structures, has to be done, when attempting to elucidate functions and functional differentiation of the cells.

In the present study, we immunocytochemically identified three clusters of neurosecretory cells, using anti-DH or -PBAN antisera, and we injected Lucifer Yellow into the cells to visualize complete structures, including neurohaemal terminals in the corpora cardiaca. Functional differentiation of DH/PBAN immunoreactive cells, identified by surgical ablation of particular clusters of cells is reported elsewhere [14].

\section{MATERIALS AND METHODS}

Animal

The female silkworms, Bombyx mori L., we used were F1 hybrids of the bivoltine races 'Kinshu' and 'Showa'. The eggs were incubated at $26^{\circ} \mathrm{C}$ under illumination. The larvae were reared on a commercially available artificial diet (Yakult Co., Japan) at $26 \pm 1^{\circ} \mathrm{C}$ with a photoperiod of $16 \mathrm{~L}: 8 \mathrm{D}$. Pupae and adults were maintained under the same conditions.

Lucifer Yellow filling

The head of a 5 th instar larva was isolated and mounted in an experimental chamber using beeswax. The chamber was filled with physiological saline designed to mimic extracellular conditions within 
the brain [25]. The SG was exposed by removing surrounding tissues. A pair of insect pins was placed under the SG of a larva to provide a supporting platform for the larval brain. The head of a pupa or a pharate adult was isolated and mounted, ventral or frontal side up, in the chamber. The SG was exposed by removing part of the cuticle over the ganglion. After incubation for $10-20 \mathrm{~s}$ in saline containing $1 \%$ pronase (Kaken Seiyaku Co. Japan), the sheath of the SG was partially removed, using a fine tungsten needle.

Under visual guidance, a soma of a neurosecretory or nonneurosecretory cell located around the ventral midline of the SG was penetrated with a bevelled glass microelectrode filled with $10 \%$ Lucifer Yellow CH (Sigma). An indifferent electrode was placed in the chamber. The microelectrode was connected to a preamplifier (MEZ-8201, Nihon Kohden), which allowed simultaneous recording and current injection via a bridge circuit. An action potential was induced from the soma by passing a depolarizing current pulse (3-4 $\mathrm{nA}, 3 \mathrm{~ms}$ in duration). Intracellular action potentials and signals for injected currents were displayed and stored on a memory oscilloscope (VC-10, Nihon Kohden).

Lucifer Yellow (LY) was iontophoretically injected by passing a hyperpolarizing current $(3 \mathrm{nA})$ for 5-10 min. Subsequently the preparation was maintained at room temperature $\left(19-23^{\circ} \mathrm{C}\right)$ for $1 \mathrm{hr}$. The tissues containing the dye were dissected out together with parts of associated structures (e.g. aorta and oesophagus) and fixed with $4 \%$ formaldehyde in $0.15 \mathrm{M}$ sodium phosphate buffer $(\mathrm{pH} 7.4$ ) for 30 $\min$. The tissues were then washed for $30 \mathrm{~min}$ in the buffer, dehydrated, cleared with methyl salicylate and gently compressed under the weight of a coverslip or by a finger. The preparations were viewed and photographed immediately at various focal planes, using a fluorescence photomicroscope (Nikon). The structure of LY-filled cells was reconstructed from photographic films, using a photographic enlarging projector.

\section{Antisera production}

Two peptides corresponding to the amino-terminal 12-amino acid sequence of $\mathrm{DH}$ and the amino-terminal 15-amino acid sequence of PBAN, both added a cysteine at the carboxyl terminus, were synthesized at The University of Tokyo. These peptides were conjugated with bovine serum albumin (BSA). Six female BALB/c mice were immunized at 2-3 week intervals by giving an intraperitoneal injection of DH(1-12)-Cys-BSA (20 $\mu \mathrm{g} /$ mouse) in Freund's complete adjuvant. Three days after each injection, the mice were bled from the tail vein and the blood was centrifuged. Antibody detection in each mouse serum was carried out by dotimmunobinding assay using the synthetic DH(1-12)-Cys. After injecting the antigen five times, the antibody activity of all mice was detected at 6,000-fold dilution of the antiserum. An antibody for PBAN(1-15)-Cys-BSA was developed by immunizing a rabbit. After injecting $0.4 \mathrm{mg}$ of antigen four times at 2 week intervals, the antibody was detected at 5,000-fold dilution of the antiserum.

\section{Immunocytochemistry}

Larval SGs were isolated and the ventral sheath of the ganglions was partially removed. The tissues were fixed in Bouin's solution for $2 \mathrm{hr}$ at room temperature, then were rinsed for $15 \mathrm{hr}$ in phosphate-buffered saline containing $0.2 \%$ Triton X-100 (PBT, pH 7.4) and incubated at $4^{\circ} \mathrm{C}$ for 3 days in rabbit anti-PBAN antiserum diluted $1: 250$ in PBT containing $0.25 \%$ BSA. These tissues were then washed in PBT at room temperature and soaked for $2 \mathrm{hr}$ in rhodamine-labeled goat anti-rabbit IgG antiserum diluted 1:40 in PBT. Next, they were washed in phosphate-buffered saline (PBS), dehydrated and cleared with methyl salicylate. Immunoreactivity of some LY-filled cells was examined in whole mount preparations stained using the same immunocytochemical method described above. The primary and secondary antisera were sometimes replaced with mouse anti-DH and rhodamine-labeled rabbit antimouse IgG antisera, respectively.

DH/PBAN immunoreactive cells of a pupa and an adult moth were usually examined in sectioned preparations because of relatively strong autofluorescence of the SG after pupation. The isolated brain-SG complex was placed in the fixative, dehydrated, soaked in xylene and embedded in paraffin. Serial sections were deparaffinized, rehydrated, washed in PBT and preincubated with PBT containing 10\% normal goat serum for $1 \mathrm{hr}$. These sections were then incubated in anti-PBAN (or anti-DH) antiserum overnight at $4^{\circ} \mathrm{C}$. The primary antiserum was diluted $1: 500$ in PBT. The sections were then rinsed in PBS and exposed to a rhodamine-labeled goat anti-rabbit (or anti-mouse) IgG antiserum diluted $1: 30$ for $1 \mathrm{hr}$. After another rinse in PBS, the sections were mounted with glycerol. Sections were sometimes double-labeled using two primary antisera (rabbit anti-PBAN and mouse anti-DH antisera) and two secondary antisera labeled with fluorescein or rhodamine.

For controls concerning the specificity of the immunocytochemical procedure, sections were incubated with the primary antisera inactivated by adding synthetic PBAN-I or DH $(1 \mu \mathrm{g} / \mathrm{ml})$. Immunostaining was almost totally abolished, though in some preparations the somata were faintly stained.

\section{RESULTS}

Gross anatomy of brain-SG-retrocerebral neurohaemal organs

Anatomical profile of the brain and SG of the larva appears markedly different from that of the adult as there are gross metamorphic changes in the CNS during adult development. Larval SG is linked to the brain by a pair of long connectives, the circumoesophageal connective, while adult $\mathrm{SG}$ is completely fused with the tritocerebral lobe of the brain (Fig. 1). However, all of the major nerve branches associated with the retrocerebral neurohaemal organs, the corpus cardiacum (CC) and the corpus allatum (CA), remain through metamorphosis, despite extensive restructuring of central and peripheral nervous system in the head. The CC-CA complex is connected to the brain by two nerves, nervus corporis cardiaci (NCC) $1+2$ and NCC 3 and is connected to the SG by NCC-ventralis (NCC-V) and two branches of the maxillary nerve ( $a$ and $b$ in Fig. 1). Many thin branches of the maxillary nerve innervating larval muscles in the maxilla appear to be greatly diminished or entirely lost at pupation as the result of changes in target muscles during development. There is a small ellipsoidal node at the point where the two branches of the maxillary nerve join together into the NCC-V. A thin nerve (NCC-RN) extending medially from the $\mathrm{CC}$ joins the recurrent nerve.

\section{Localization of DH/PBAN immunoreactive cells}

Twelve neurosecretory cells in the SG showed potent immunoreactivity to the rabbit anti-PBAN antiserum. In larva, the somata are localized at three distinct regions, as clusters on the ventral midline of the SG (Fig. 2A). The 

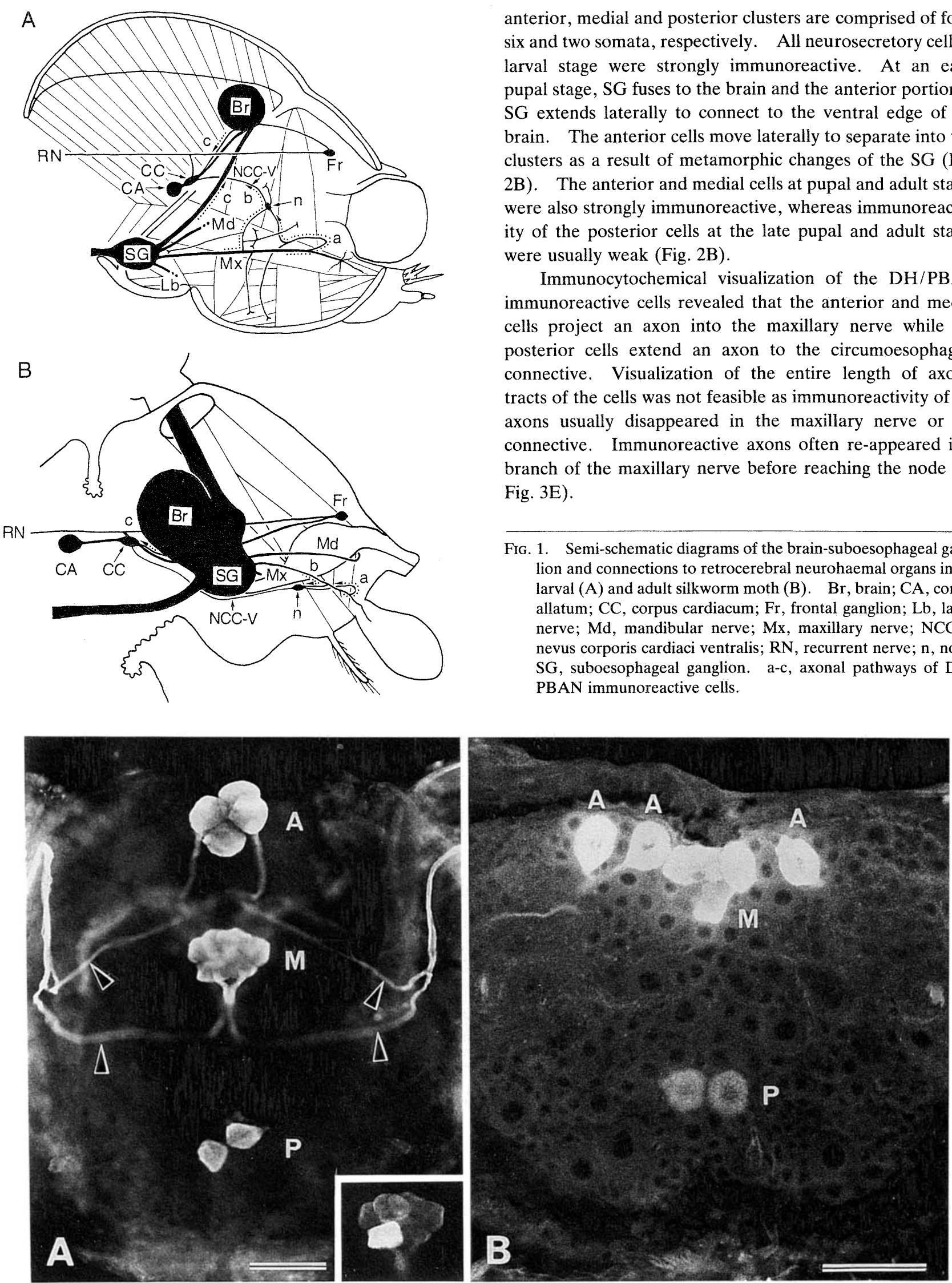

Fig. 2. Ventral views of PBAN immunoreactive cells' somata in the larval (A) and adult SG (B). A, anterior cell (cluster); M, medial cell cluster; P, posterior cell cluster. Bundles of immunoreactive axonal processes (arrowheads) are seen, though they are out of focus in this picture. Inset illustrates a medial cell that has been intracellularly injected with Lucifer Yellow. Scale bars, $100 \mu \mathrm{m}$. anterior, medial and posterior clusters are comprised of four, six and two somata, respectively. All neurosecretory cells at larval stage were strongly immunoreactive. At an early pupal stage, SG fuses to the brain and the anterior portion of SG extends laterally to connect to the ventral edge of the brain. The anterior cells move laterally to separate into two clusters as a result of metamorphic changes of the SG (Fig. 2B). The anterior and medial cells at pupal and adult stages were also strongly immunoreactive, whereas immunoreactivity of the posterior cells at the late pupal and adult stages were usually weak (Fig. 2B).

Immunocytochemical visualization of the DH/PBAN immunoreactive cells revealed that the anterior and medial cells project an axon into the maxillary nerve while the posterior cells extend an axon to the circumoesophageal connective. Visualization of the entire length of axonal tracts of the cells was not feasible as immunoreactivity of the axons usually disappeared in the maxillary nerve or the connective. Immunoreactive axons often re-appeared in a branch of the maxillary nerve before reaching the node (cf. Fig. 3E).

Frg. 1. Semi-schematic diagrams of the brain-suboesophageal ganglion and connections to retrocerebral neurohaemal organs in the larval (A) and adult silkworm moth (B). Br, brain; CA, corpus allatum; CC, corpus cardiacum; Fr, frontal ganglion; Lb, labial nerve; $\mathrm{Md}$, mandibular nerve; $\mathrm{Mx}$, maxillary nerve; $\mathrm{NCC}-\mathrm{V}$, nevus corporis cardiaci ventralis; $\mathrm{RN}$, recurrent nerve; $\mathrm{n}$, node; $\mathrm{SG}$, suboesophageal ganglion. a-c, axonal pathways of $\mathrm{DH} /$ PBAN immunoreactive cells. 

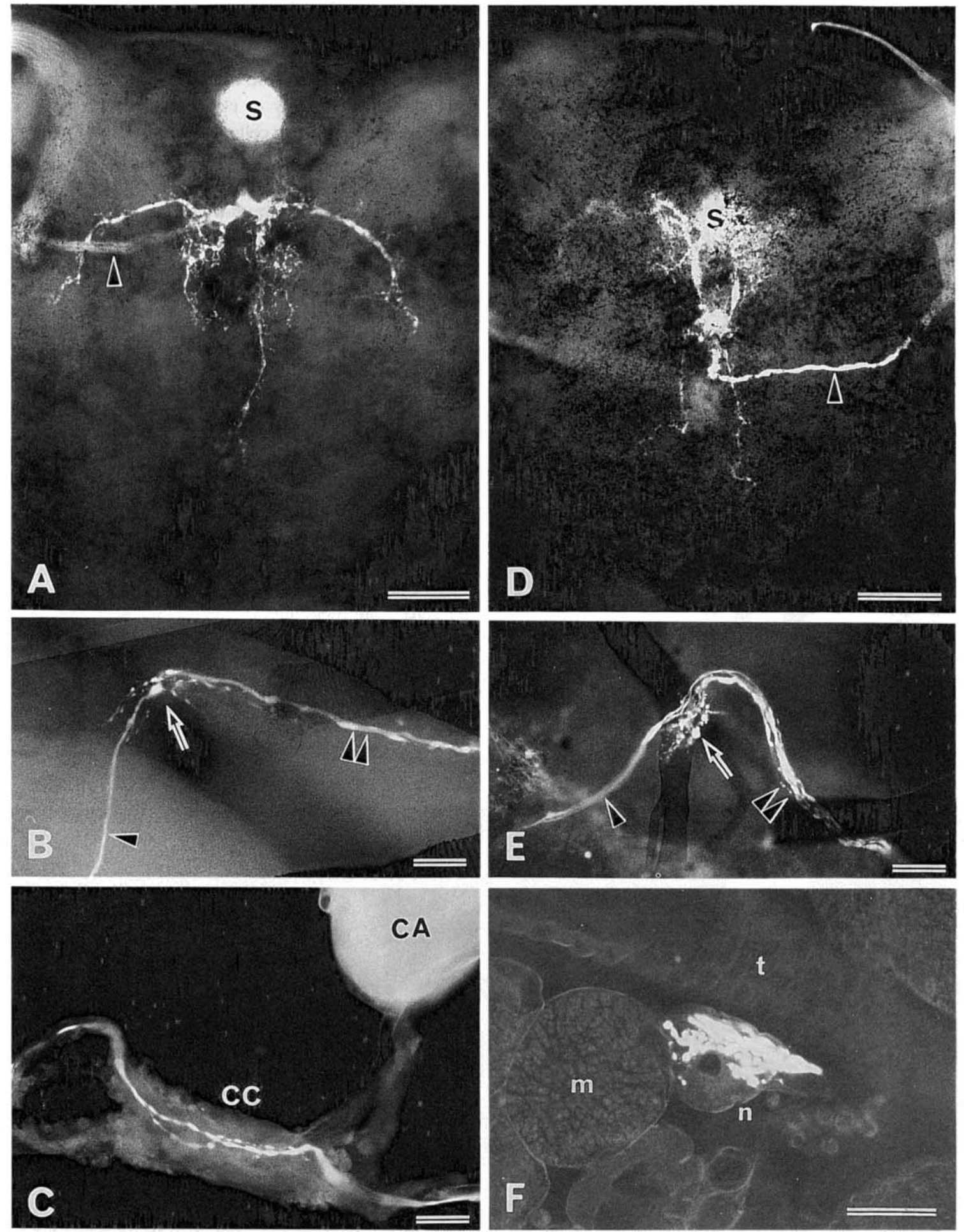

FIG. 3. Photomicrographs of an anterior and a medial cell in the larval SG visualized by injection of Lucifer Yellow (A-D) or PBAN immunocytochemistry (E, F). A. Ventral view of an anterior cell. S, a soma (out of focus); arrowhead, an axon. B. Axonal profile of the anterior cell. The axon (single arrowhead) is smooth in appearance before reaching the node (arrow), but has varicose appearance in the node and the NCC-V (double arrowheads). C. Varicose axonal processes in the corpus cardiacum (CC). CA, corpus allatum. D. Ventral view of a medial cell. S, a soma; arrowhead, an axon. E. PBAN immunoreactive axonal processes. They (single arrowhead) appear smooth before invading the node (arrow), but give rise to varicose branches in the node and the NCC-V (double arrowheads). F. Varicose branches in the node (n). m, a muscle; $t$, trachea. Scale bars, $100 \mu \mathrm{m}(\mathrm{A}, \mathrm{D}), 50 \mu \mathrm{m}(\mathrm{B}, \mathrm{C}, \mathrm{E}, \mathrm{F})$. 
Although we used mostly the anti-PBAN antiserum, similar results were obtained when the anti-DH antiserum was used as a primary antiserum. Double labeling of cells using anti-DH and anti-PBAN antisera revealed that the same three clusters of neurosecretory cells were immunoreactive with the two antisera (data not shown).

\section{Structure of DH/PBAN immunoreactive cells}

Somata of neurosecretory cells in the SG could often be distinguished by their opalescence. We impaled many opalescent somata at the three regions where DH/PBAN immunoreactive cells were expected to be present. Before injecting LY, action potentials of an impaled cell were recorded because a neurosecretory cell was discernible from a non-neurosecretory cell by large and prolonged action potentials $[12,25]$. All those neurosecretory cells present at medial and posterior regions were $\mathrm{DH} / \mathrm{PBAN}$ immunoreactive cells (inset of Fig. 2A), but cells at the anterior region were sometimes different neurosecretory cells with no immunoreactivity to the antisera and an axon projecting to the mandibular nerve.

Anterior and medial cells: Injection of LY into a soma of an anterior or a medial cell of the larva revealed that the primary neurite originating from the soma extended for some distance posteriorly or dorsally and bifurcated into dendritic processes. The dendritic processes of the anterior and medial cells are shaped " $\pi$ " (Fig. 3A) or T (Fig. 4A) and X (Figs. 3D, $4 \mathrm{~B})$, respectively. An axon originating from one of the dendritic processes extends laterally and turns anteriorly to enter the maxillary nerve contralateral to cell's soma. All seven anterior cells examined in this study had an axon that ran in the maxillary nerve toward the mandible and turned posteriorly to reach the ellipsoidal node (route a in Fig. 1A). Six out of eleven medial cells examined had such a long axon and the others had a relatively short axon that entered a shortcut branch on the halfway (route b in Fig. 1A) to reach the node. Axons of both anterior and medial cells had a few varicose branches in the node where two cells of unknown origin were present (Fig. 3B,F). Axons leaving the node passed through the NCC-V and reached the $\mathrm{CC}$ (Figs. $3 \mathrm{C}$ and 4A, B). They usually invaded into a nerve (NCC-RN) and had a varicose appearance not only in the $\mathrm{CC}$ but also in the full length of the NCC-V (Fig. 3B). Such a structural specialization is often present in immunocytochemical preparations (Fig. 3E).
A
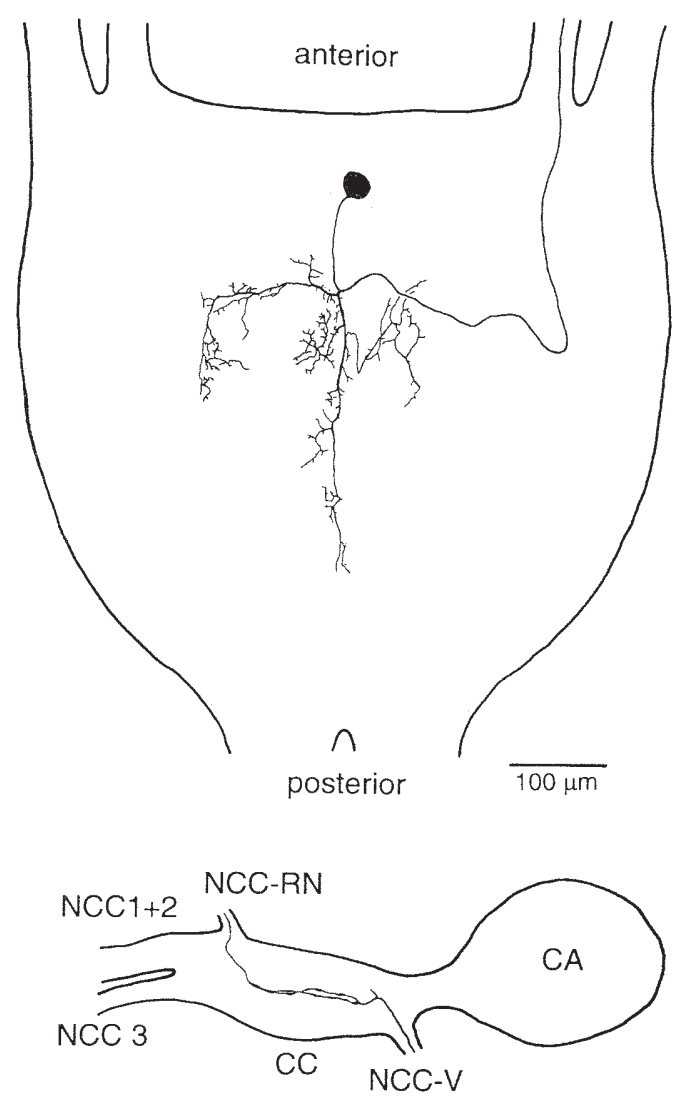

B
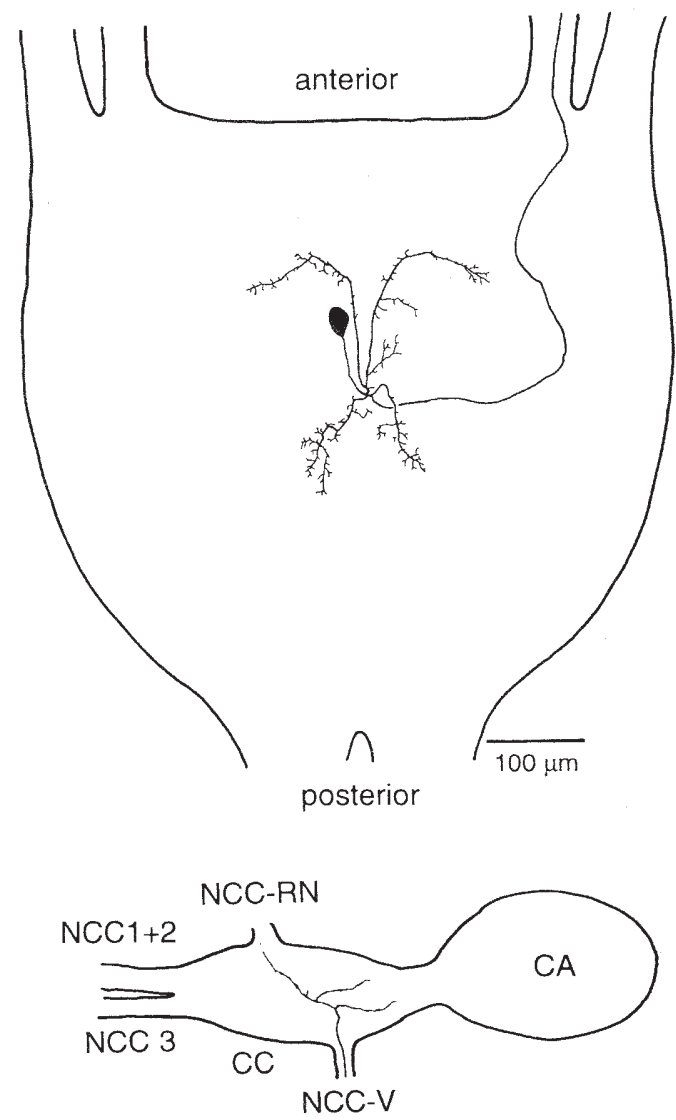

Fig. 4. Drawings of an anterior (A) and a medial cell (B) in the larval SG. CA, corpus allatum; CC, corpus cardiacum; NCC $1+2$, fused nerve of nervus corporis cardiaci 1 and 2 ; NCC 3 , nervus corporis cardiaci 3 ; NCC-V, nervus corporis cardiaci ventralis; NCC-RN, nervus corporis cardiaci recurrens that joins the recurrent nerve. 
Although the anatomical appearance of neurosecretory cells at a late pupal or an adult stage somewhat differs from that at the larval stage because of the gross anatomical change of SG during adult development, branching patterns of primary dendrites of the cells are basically similar to those of larval cells (Fig. 5). Axons of pupal or adult cells, like the larval cells, extended toward the node and NCC-V via two branches of the maxillary nerve (route $a$ and $b$ in Fig. 1B). Varicose branches of those axons could be observed in the node, but no axon could be traced to its terminal in the $\mathrm{CC}$, using the dye-filling method because of elongation of NCC-V
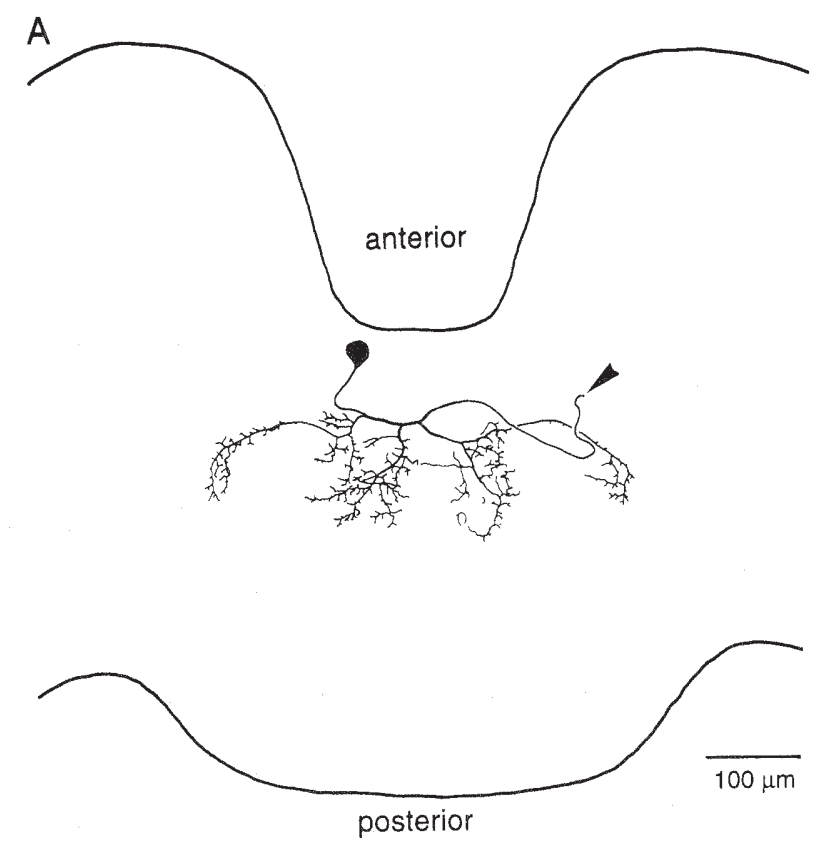

B

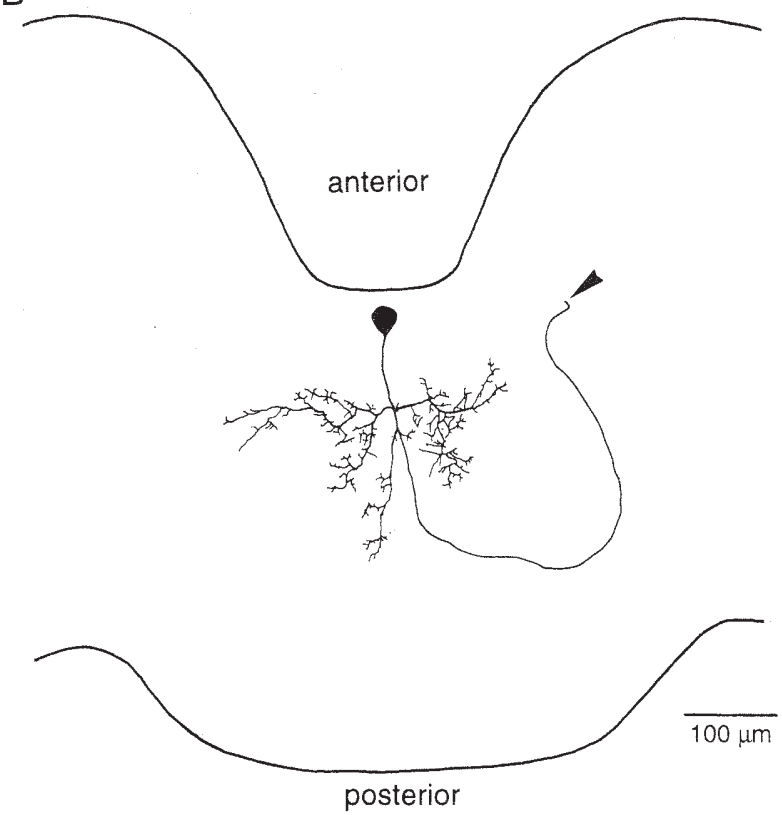

Fig. 5. Dorsal views of an anterior (A) and a medial cell (B) in adult females. Axons exit the SG into the maxillary nerve at the points indicated by arrowheads. during development.

Posterior cells: Fig. 6A shows a pair of posterior cells visualized by LY injection. Position of both somata, dendritic branches and axonal pathways are almost symmetric around the midline of the ganglion. LY injection into a single cell reveals that each cell has a symmetric dendritic abor and dendritic branches of the two cells overlap (Fig. 7A). The primary neurite extending from the soma runs toward the dorsal surface for a short distance, then turns anteriorly to bifurcate into dendritic processes. Two dendritic processes run side by side along the midline of the SG for some distance and each process turns laterally and then posteriorly to give off many fine branches. An axon originating from the primary neurite or one of the dendritic processes extends laterally and turns anteriorly to enter the circumoesophageal connective contralateral to the cell's soma. The axon, after running up the connective (route $\mathrm{c}$ in Fig. 1A) and passing through the lateral part of the brain, enters the nerves corpus cardiaci 3 (NCC 3 ) to project to the $\mathrm{CC}$, an area where the terminal branches form a complex network of numerous varicosities. A few terminal branches invaded the associated nerves of the CC, NCC-RN and NCC-V (Figs. 6B, 7B) but did not invade the corpus allatum (CA).

Figure $6 \mathrm{C}$ shows a ventral view of a posterior cell of a pupa at 4 days after pupation, a time when the brain-SG complex takes on an adult form. The bilateral dendritic branches expand to cover the anterior edge of the neuropile around the oesophageal foramen. The axon first projects postero-dorsally and finally leaves the brain posteriorly after a few turns in the brain-SG complex. The anatomical profile of the cells in the brain-SG complex at the adult stage is almost identical to that in the complex at the mid-pupal stage (Figs. 6D and 8A,B). Their axons are characterized by dense fasciculation in the $\mathrm{CC}$ and many fine, parallel branches in the recurrent nerve under the aorta (Figs. 6E and $8 \mathrm{C}$ ). A few branches invading the allatal nerve (NCA) reach the proximal portion of the corpora allata (CA) (Fig. 8C).

\section{DISCUSSION}

$\mathrm{DH}$ and PBAN of Bombyx mori are generated along with three other structurally-related peptides from a common precursor polyprotein that is translated from a single species of mRNA [16, 30]. In situ hybridization with cDNA encoding the precursor polyprotein revealed twelve neurosecretory cells that localize somata as clusters on the ventral side of three different neuromeres of SG: mandibular, maxillary, and labial neuromeres [31]. Since the number, size and position of neurosecretory cells we visualized immunocytochemically are identical to those of the cells revealed by cDNA hybridization, it is apparent that the anterior, medial and posterior DH/PBAN immunoreactive cells correspond to the neurosecretory cells in the mandibular, maxillary and labial neuromeres, respectively. It remains to be determined if all the neurosecretory cells produce functional 

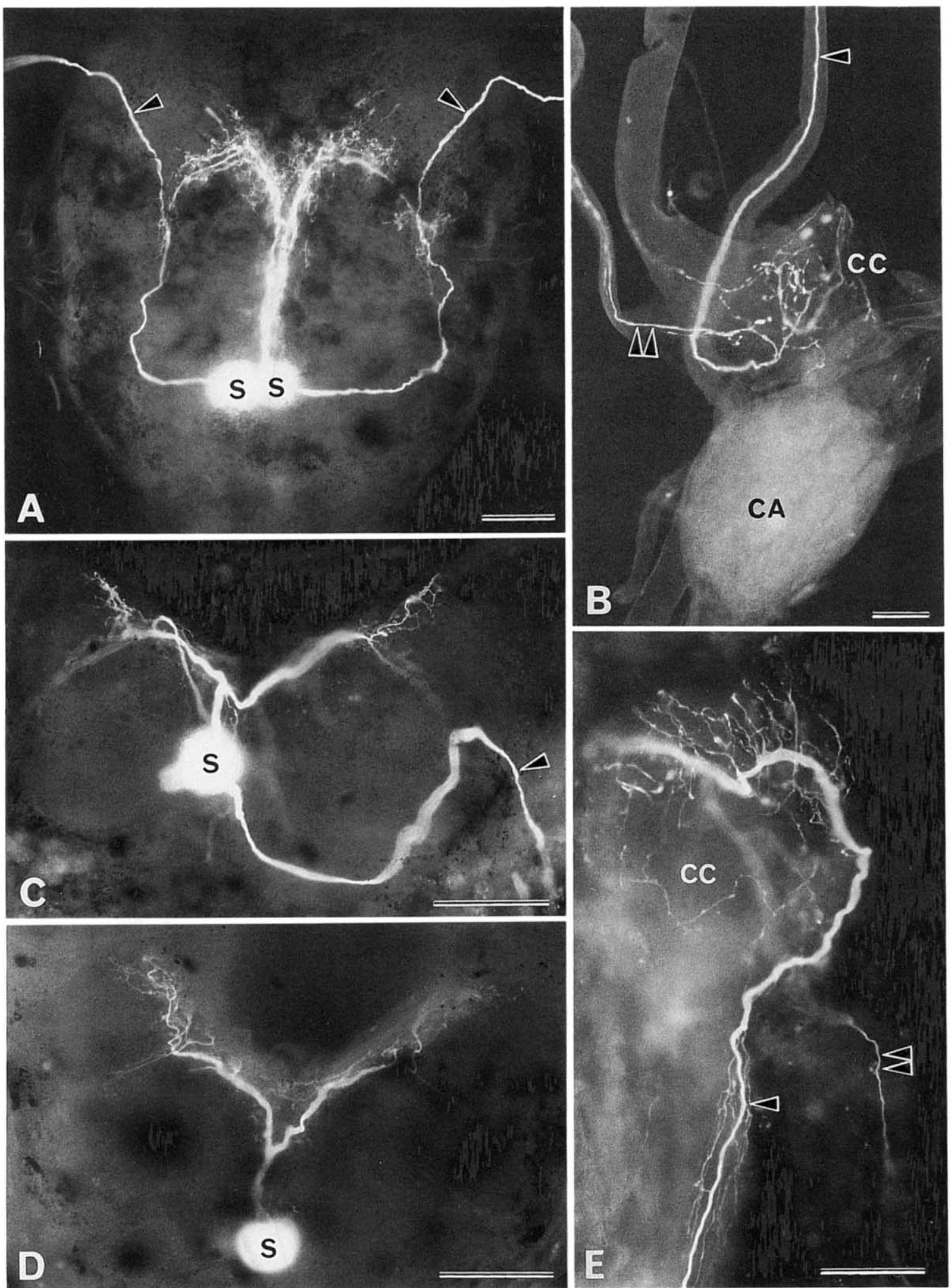

FIg. 6. Photographs of posterior cells in the SG of a 5th instar larva (A,B), a 4-day old pupa (C) and an adult moth (D,E). A. Ventral view of larval SG. S, a soma (out of focus); arrowhead, an axon. B. Dorsal view of larval neurohaemal organs, the corpus cardiacum (CC) and the corpus allatum (CA). arrowhead, a smooth axon in the NCC 3; double arrowheads, varicose axonal branches projecting to the recurrent nerve. C. Ventral view of pupal SG. S, a soma (out of focus); arrowhead, an axon. D. Frontal view of adult SG. S, a soma. E. Ventral view of adult corpus cardiacum (CC). arrowhead, an axonal process invading the recurrent nerve; double arrowheads, an axonal branch in the allatal nerve. Scale bars, $100 \mu \mathrm{m}(A, C, D)$ or $50 \mu \mathrm{m}(\mathrm{B}, \mathrm{E})$. 
A

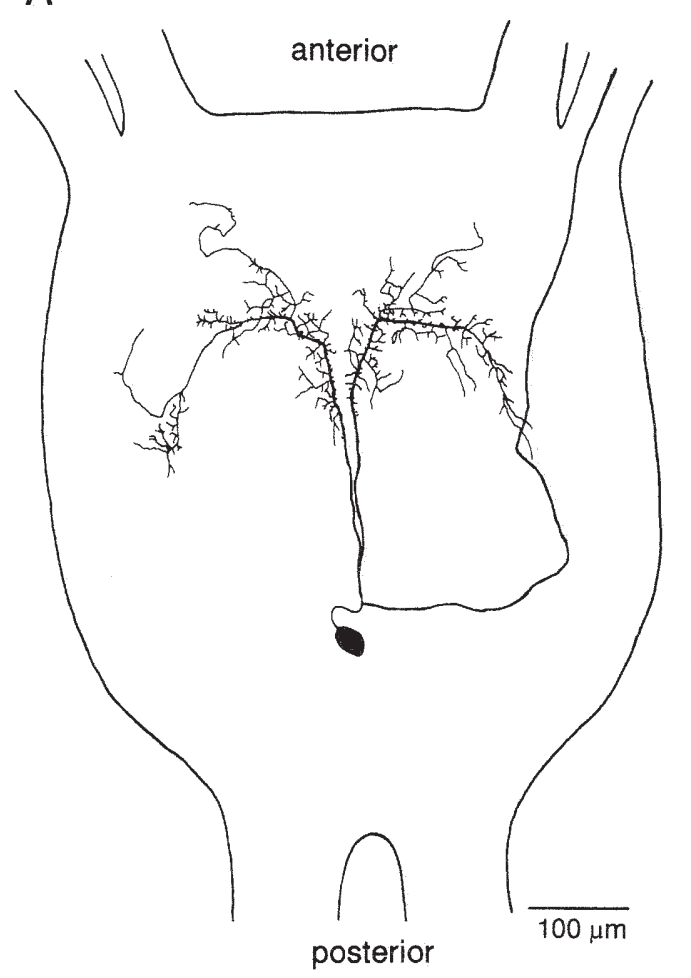

B

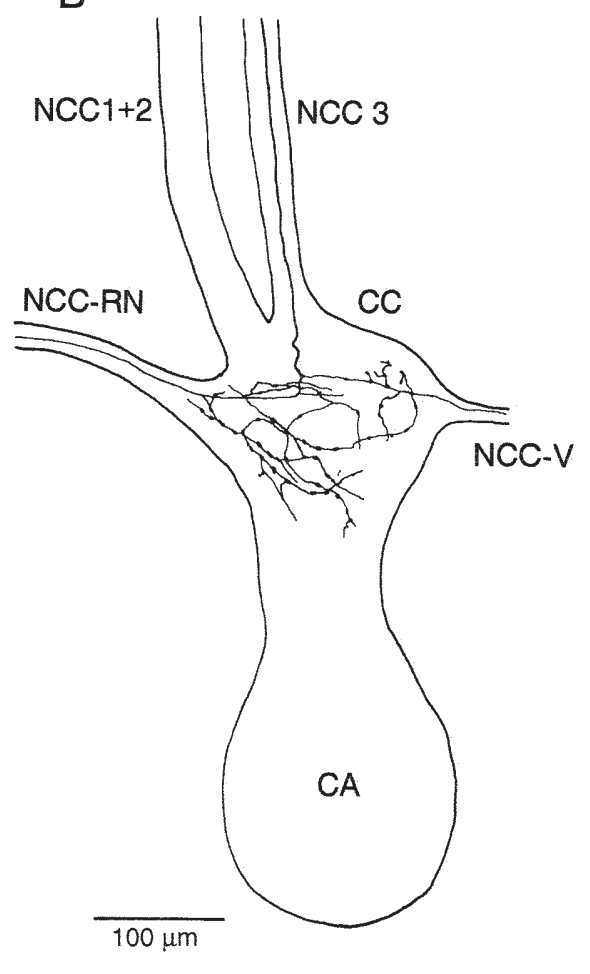

FIG. 7. A. Ventral view of a posterior cell in a 5th instar larva. B. Dorsal view of an axon terminal of the cell. Abbreviations are the same as for Fig. 4.
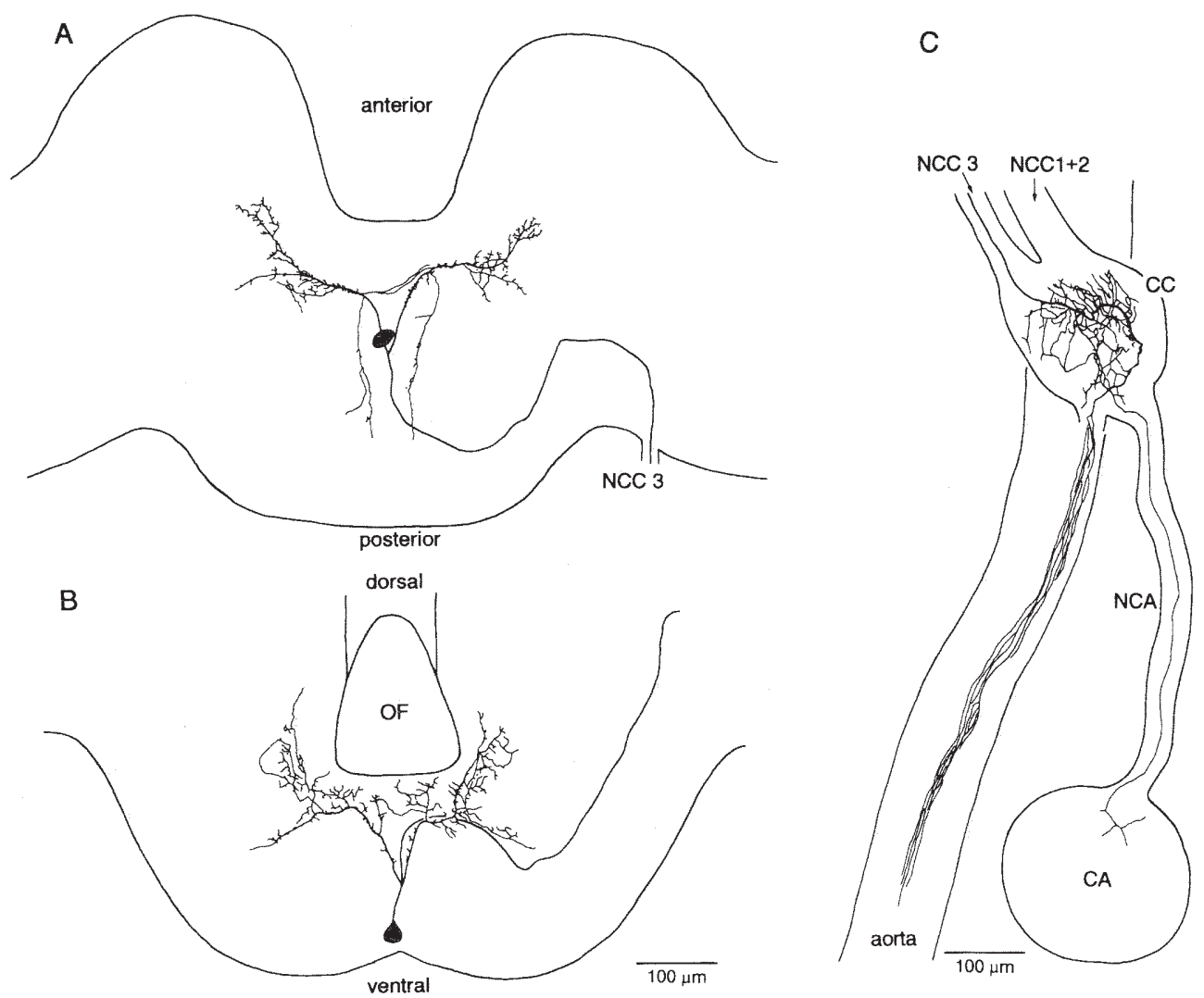

FIG. 8. Ventral (A) and frontal view (B) of a posterior cell and its axon terminal (C) of an adult female. OF, oesophageal foramen; CC, corpus cardiacum; CA, corpus allatum; NCC 1+2, nervi corporis cardiaca 1 and 2; NCC 3, nervus corporis cardiaci 3; NCA, nervus corporis allati. 
(mature) DH and/or PBAN and secrete these into the haemolymph at appropriate stages of postembryonic development. Specific morphological profiles of the three sets of cells revealed by injection of LY suggests that they may be under the control of self neural mechanisms to play specific physiological functions. Some evidence for such a functional specialization has been obtained from a surgical experiment showing that potent $\mathrm{DH}$ activity is responsible for the posterior cells while potent pheromonotropic activity is linked to the medial cells [14]. However, functional specialization of cells does not simply mean that they produce a specific neuropeptide, because five neuropeptides generated from the precursor usually have significant $\mathrm{DH}$ and pheromonotropic activities [30].

Since a group of FXPRLamide peptides, including DH and PBAN, is widely distributed among insects and can regulate many physiological processes [22-24], DH/PBAN immunoreactive cells reported here may be involved in regulation of other processes besides induction of embryonic diapause and pheromone production. The neurosecretory cells are strongly immunoreactive at the larval stage (Fig. 2A) as well as at the pupal and adult stages of the female when $\mathrm{DH}$ and PBAN function, respectively (Fig. 2B). In a preliminary experiment, similar DH/PBAN immunoreactive cells were also found in males at the larval, pupal and adult stages. These distributions of DH/PBAN immunoreactivity suggest a multiplicity of functions.

Many FXPRLamide peptides have a myotropic activity $[11,20,26,27]$. The anterior and medial cells have numerous varicosities (putative neurohaemal structure) in the NCC$\mathrm{V}$ which pass closely between musculatures of the mandible and the maxilla (Fig. 3B, E), though they showed no significant sign of direct innervation into particular muscles of the mouthparts. Thus, neurohormones released from the nerve may act locally on the musculatures. FXPRLamide peptides have an effect on cuticular melanization and epidermal reddish brown pigmentation of some species of armyworms $[23,24]$. Because PBAN-I of Bombyx mori can produce strong melanization of cuticle of Psudaletia separata, the neuropeptide and other structurally-related neuropeptides from Bombyx mori may be involved in regulating integumental pigmentation of the silkworm. The anterior and medial cells have a few varicose branches in the small node (Fig. 3B, E, F), thereby suggesting that two cells present in the node could be possible targets of their modulatory actions. Although origin and functional nature of the cells are unknown, these may be a kind of peripheral (neurosecretory) neuron localized in the course of peripheral nerves [3, 4 , $8,10,29]$. Some peripheral neurons appear to function as a monitor for changes in the internal environment such as concentrations of amino acids and sugars in the haemolymph [28].

Fukuda and Takeuchi [6] found a pair of neurosecretory cells with an azocarminophilic material at the particular area in the ventral side of presumptive labial neuromere of SG and these were regarded as being putative diapausing factor producing cells, because the amount of the neurosecretory material apparently differed between a diapausing-egg producer and a non-diapausing producer [7]. It is apparent that these neurosecretory cells correspond to posterior $\mathrm{DH} /$ PBAN immunoreactive cells, because in the present study we detected no other putative neurosecretory cell with a prolonged action potential and located around the posterior cells. Axons extending from the posterior cells pass the brain and reach the CC (Figs. 7, 8). If the cells produce DH in the somata and transport it to the $\mathrm{CC}$ along such an axonal pathway, the pathway would provide an anatomical basis for the distribution of DH activity obtained from the brain and the $\mathrm{CC}[32,33]$.

PBAN immunocytochemistry revealed three clusters of neurosecretory cells in the same regions of the SG of Helicoverpa zea, though the number of cells comprising each cluster differs from that of Bombyx mori reported here [17]. However, the medial (maxillary) cluster contains two pair of cells that project an axon into the ventral nerve cord (VNC) and which terminate in the terminal abdominal ganglion (TAG). This axonal pathway seems to be involved in the transportation of PBAN and/or PBAN-like peptides, because an intact $\mathrm{VNC}$ is needed for normal pheromone production [34]. The peptides transported to the TAG appear to be released into the ganglion and stimulate aminergic neurons innervating pheromone glands to produce sex pheromone [2, 34]. In Bombyx mori, there was no cell with such an axon descending the VNC, thereby suggesting that pheromonotropic peptides produced in the somata may be transported to the $\mathrm{CC}$ via branches of the maxillary nerve and the NCC-V, then are released into the haemolymph to act directly on pheromone glands.

DH/PBAN immunoreactive cells send their axons to the $\mathrm{CC}$ through specific axonal pathways. The anterior and medial cells' axons in particular project into the peripheral nerve that runs under the cuticle of the pupal and adult head (Fig. 1B). Secretion of pheromonotropic peptides is assigned to the medial and anterior cells [14], and seems to be completely inhibited at a critical period of time after the initiation of copulation [13]. These characteristic features of the neurosecretory system make this system an interesting model suitable for electrophysiological analyses of secretory activities of neurosecretory cells at a particular stage of development and control mechanisms of secretion in insects.

\section{ACKNOWLEDGMENTS}

We thank M. Ohara for helpful comments on the manuscript. This work was supported in part by the Grant-in-Aid from the Ministry of Education, Science and Culture of Japan.

\section{REFERENCES}

1 Ando T, Arima R, Uchiyama M, Nagasawa $H$, Inoue T, Suzuki A (1988) Pheromone biosynthesis activating neuropeptide hormone in the head of the silkworm moth. Agric Biol Chem 52: 881-883 
2 Christensen TA, Itagaki H, Teal PEA, Jasensky RD, Tumlinson JH, Hildebrand JG (1991) Innervation and neural regulation of the sex pheromone gland in female Heliothis moths. Proc Natl Acad Sci USA 88: 4971-4975

3 Davis NT (1987) Neurosecretory neurons and their projections to the serotonin neurohemal system of the cockroach Periplaneta americana (L.), and identification of mandibular and maxillary motor neurons associated with this system. J Comp Neurol 259: 604-621

4 Finlayson LH, Osborne MP (1968) Peripheral neurosecretory cells in the stick insect (Carausius morosus) and the blowfly larva (Phormia terrae-novae) J Insect Physiol 14: 1793-1801

5 Fukuda S (1951) Factors determining the production of the non-diapause eggs in the silkworm. Proc Japan Acad 27: 582586.

6 Fukuda S, Takeuchi S. (1967a) Diapause factor producing cells in the suboesophageal ganglion of the silkworm, Bombyx mori L. Proc Japan Acad 43: 51-56.

7 Fukuda S, Takeuchi S (1967b) Studies on the diapause factorproducing cells in the suboesophageal ganglion of the silkworm, Bombyx mori L. Embryologia 9: 333-353.

8 Griffiths AC, Finlayson LH (1982) Extra-ganglionic neurosecretory and non-neurosecretory neurons in the larva of the moth, Agrotis segetum Schiff (Lepidoptera: Noctuidae). Int J Insect Morphol Embryol 11: 341-349

9 Hasegawa K (1951) Studies on the voltinism in the silkworm, Bombyx mori L., with special reference to the organs concerning determination on voltinism. Proc. Japan Acad. 27, 667-671.

10 Hinks CF (1975) Peripheral neurosecretory cells in some Lepidoptera. Can J Zool 53: 1035-1038

11 Holman GM, Nachman RJ, Wright MS (1990) Insect neuropeptide. Annu Rev Entomol 35: 201-217

12 Ichikawa $\mathrm{T}$ (1991) Architecture of cerebral neurosecretory cell systems in the silkworm Bombyx mori. J exp Biol 161: 217237.

13 Ichikawa T, Shiota T, Kuniyoshi H (1996) Neural inactivation of sex pheromone production of mated females of the silkworm moth, Bombyx mori. Zool Sci (in press)

14 Ichikawa T, Shiota T, Shimizu I, Kataoka H (1996) Functional differentiation of neurosecretory cells with immunoreactive diapause hormone and pheromone biosynthesis activating neuropeptide of the silkworm, Bombyx mori. Zool Sci (in press)

15 Imai K, Konno T, Nakazawa Y, Komiya T, Isobe M, Koga K, Goto T, Yaginuma T, Sakakibara K and Yamashita O (1991) Isolation and structure of diapause hormone of the silkworm, Bombyx mori. Proc Acad Japan B 67: 98-101

16 Kawano $\mathrm{T}$, Kataoka $\mathrm{H}$, Nagasawa $\mathrm{H}$, Isaogai $\mathrm{A}$, and Suzuki $\mathrm{A}$ (1992) cDNA cloning and sequence determination of the pheromone biosynthesis activating neuropeptide of the silkworm, Bombyx mori. Biochem Biophys Res Comm 189: 221-226

17 Kingan TG, Blackburn MB and Raina AK (1992) The distribution of pheromone-biosynthesis-activating neuropeptide (PBAN) immunoreactivity in the central nervous system of the corn earworm moth, Helicoverpa zea. Cell Tissue Res 270: 229-240

18 Kitamura A, Nagasawa $\mathrm{H}$, Kataoka $\mathrm{H}$, Inoue $\mathrm{T}$, Matsumoto $\mathrm{S}$, Ando T, Suzuki A (1989) Amino acid sequence of pheromonebiosynthesis-activating neuropeptide (PBAN) of the silkworm, Bombyx mori. Biochem Biophys Res Commun 163: 520-526

19 Kitamura A, Nagasawa H, Kataoka H, Ando T, Suzuki A (1990) Amino acid sequence of pheromone biosynthesis activating neuropeptide-II (PBAN-II) of the silkmoth, Bombyx. mori. Agric Biol Chem 54: 2495-2497
20 Kuniyoshi H, Nagasawa H, Ando T, Suzuki A (1992) Crossactivity between pheromone biosynthesis activating neuropeptide (PBAN) and myotropic pyrokinin insect peptides. Biosci Biotec Biochem 56: 167-168

21 Ma PWK, Knipple DC, Roelofs WL (1994) Structural organization of the Helicoverpa zea gene encoding the precursor protein for pheromone biosynthesis-activating neuropeptide and other neuropeptides. Proc Natl Acad Sci USA 91: 6506-6510

22 Matsumoto S, Fonagy A, Kurihara M, Uchiumi K, Nagamine T, Chijimatsu M, Mitsui T (1992) Isolation and primary structure of a novel pheromonotropic neuropeptide structurally related to leucopyrokinin from the armyworm larvae, Pseudaletia separata. Biochem Biophys Res Commun 182: 534-539

23 Matsumoto S, Kitamura A, Nagasawa H, Kataoka H, Orikasa C, Matsui T, Suzuki A (1990) Functional diversity of a neurohormone produced by the suboesophageal ganglion: molecular identity of melanization and reddish colouration hormone and pheromone biosynthesis activating neuropeptide. J Insect Physiol 36: 427-432

24 Matsumoto S, Yamashita O, Fonagy A, Kurihara M, Uchiumi K, Nagamine T, Mitsui T (1992) Functional diversity of a pheromonotropic neuropeptide: induction of cuticular melanization and embryonic diapause in lepidopteran insects by Pseudaletia pheromonotropin. J Insect Physiol 38: 847-851

25 Miyazaki S (1980) The ionic mechanism of action potentials in neurosecretory cells and non-neurosecretory cells of the silkworm. J comp Physiol 140: 43-52.

26 Nachman RJ, Holman GM, Schoofs L, Yamashita O (1993) Silkworm diapause induction activity of myotropic pyrokinin insect neuropeptides. Peptide 14: 1043

27 Nachman RJ, Roberts VA, Dyson HJ, Holman GM, Tainer JA (1991) Active conformation of an insect neuropeptide family. Proc Natl Acad Sci USA 88: 4518-4522

28 Okajima A, Kumagai K, Watanabe M (1989) The involvement of intraceptive chemosensory activity in the nervous regulation of the prothoracic gland in a moth, Mamestra brassicae. Zool Sci 6: 859-866

29 Okajima A, Watanabe M (1989) Electrophysiological identification of neuronal pathway to the prothoracic gland and the change in electrical activities of the prothoracic gland innervating neurons during larval development of a moth, Mamestra brassicae. Zool Sci 6: 459-468

30 Sato $\mathrm{Y}$, Oguchi $\mathrm{M}$, Menjo N, Imai K, Saito $\mathrm{H}$, Ikeda M, Isobe M, Yamashita O (1993) Precursor polyprotein for multiple neuropeptides secreted from the suboesophageal ganglion of the silkworm Bombyx mori: Characterization of the cDNA encoding the diapause hormone precursor and identification of additional peptides. Proc Natl Acad Sci USA 90: 3251-3255

31 Sato Y, Ikeda M, Yamashita O (1994) Neurosecretory cells expressing the gene for common precursor for diapause hormone and pheromone biosynthesis-activating neuropeptide in the suboesophageal ganglion of the silkworm, Bombyx mori. Gen Com Endocrinol 96: 27-36

32 Takeda S (1976) Stage dependency of diapause egg production by corpora cardiaca and corpora allata complex of the silkworm, Bombyx mori (Lepidoptera: Bombycidae). App Ent Zool 12: $80-82$.

33 Takada S, Ogura N (1976) Induction of egg diapause by implantation of corpora cardiaca and corpora allata in Bombyx mori. J Insect Physiol 22: 941-944

34 Teal PEA, Tumlinson JH, Oberlander H (1989) Neural regulation of sex pheromone biosynthesis in Heliothis moths. Proc Natl Acad Sci USA 86: 2488-2492 